\title{
ANAESTHETIC MANAGEMENT IN A CASE OF PERIPARTUM CARDIOMYOPATHY AND SEIZURE DISORDER- A CASE REPORT
}

\author{
Tushar B. Munnoli1, Shraddha Naik2, Sunil Khyadi3, Gagandeep Singh ${ }^{4}$, Naveen Kumar Naveen ${ }^{5}$ \\ ${ }_{1}^{1}$ Postgraduate Student, Department of Anaesthesia, Krishna Institute of Medical Sciences, Karad. \\ ${ }^{2}$ Assistant Professor, Department of Anaesthesia, Krishna Institute of Medical Sciences, Karad. \\ 3 Senior Resident, Department of Anaesthesia, Krishna Institute of Medical Sciences, Karad. \\ ${ }_{4}^{4}$ Postgraduate Student, Department of Anaesthesia, Krishna Institute of Medical Sciences, Karad. \\ ${ }_{5}^{5}$ Postgraduate Student, Department of Anaesthesia, Krishna Institute of Medical Sciences, Karad.
}

\section{ABSTRACT}

\section{BACKGROUND}

Dilated cardiomyopathy (DCM) is a form of cardiomyopathy characterised by left ventricular or biventricular dilatation and impaired ventricular contractility. Peripartum cardiomyopathy (PPCM) is a disease affecting the parturient during late pregnancy or immediately after delivery. It has an incidence of one per 3500 live births and is associated with a high mortality rate (30-60\%).[1] The major concern while managing these patients is to optimise fluid administration and avoid myocardial depression, while maintaining stable intraoperative haemodynamics. Seizure disorders are $2^{\text {nd }}$ most prevalent and most serious neurological conditions encountered in pregnant women after migraine. Epilepsy can affect the course of pregnancy, labour, delivery and alter the foetal development whereas pregnancy can exacerbate epilepsy.[2,3] Metabolism of antiepileptic medications during pregnancy is changed and teratogenic effects of several anticonvulsant medications are unquestioned. Anaesthetic management of such cases poses a challenge due to the increased risk of various perioperative complications. We report the successful anaesthetic management of a patient with PPCM and seizure disorder posted for Suction Evacuation with Sterilisation.

\section{KEYWORDS}

Anaesthesia, Peripartum Cardiomyopathy, Seizure Disorder, Perioperative Complications.

HOW TO CITE THIS ARTICLE: Munnoli TB, Naik S, Khyadi S, et al. Anaesthetic management in a case of peripartum cardiomyopathy and seizure disorder- A case report. J. Evolution Med. Dent. Sci. 2016;5(89):6661-6663, DOI: 10.14260/jemds/2016/1506

\section{BACKGROUND}

Despite the advances in management of heart disease, pregnancy puts the mother at risk. The risk is so great with some cardiovascular abnormalities that a recommendation of interruption of pregnancy is supportable.

Peripartum cardiomyopathy 4 is a dilated cardiomyopathy associated with cardiac failure in the last month of pregnancy or within five months of delivery. This results in systolic dysfunction of the heart with decreased left ventricular ejection fraction and progressive congestive cardiac failure. There is an increased risk of malignant arrhythmias, thromboembolism and even sudden death. Pregnancy in patients with pre-existing DCM can flare up the disease and can be fatal.

In 1997, the National Heart, Lung and Blood Institute and the Office of Rare Diseases commenced the workshop that established the following diagnostic criteria for peripartum cardiomyopathy:

1. Development of Cardiac failure in the last month of pregnancy or within 5 months after delivery.

2. Absence of an identifiable cause for the cardiac failure.

3. Absence of recognisable heart disease prior to the last month of pregnancy.

Financial or Other, Competing Interest: None.

Submission 30-09-2016, Peer Review 27-10-2016,

Acceptance 02-11-2016, Published 07-11-2016.

Corresponding Author:

Dr. Tushar B. Munnoli,

Room No. 44,

IHR Hostel,

KIMS, Malkapur,

Karad-415110.

E-mail: tusharmunnoli@gmail.com

DOI: $10.14260 /$ jemds/2016/1506

\section{(c) (1) $(9)$}

4. Left ventricular systolic dysfunction demonstrated by classic echocardiographic criteria such as depressed shortening fraction or ejection fraction.

Pregnancy with seizure disorder is considered high risk mainly due to teratogenic potential of antiepileptic drugs and increased risk of pregnancy and neonatal complications i.e. hypertension, preeclampsia, antepartum haemorrhage, caesarean delivery, still births, neonatal deaths, intrauterine growth retardation and preterm delivery compared with general obstetric population. Metabolism of antiepileptic medications during pregnancy is changed and teratogenic effects of several anticonvulsant medications are unquestioned.

Anaesthetic management of such cases poses a challenge due to the increased risk of various perioperative complications. We report the successful anaesthetic management of a patient with PPCM and Seizure disorder posted for Suction Evacuation and sterilisation.

\section{CASE REPORT}

A 24-year-old booked (Gravida 3, Para 1, Live 1) with 9 weeks' gestation and a known case of Peripartum Cardiomyopathy and seizure disorder was scheduled for elective Suction Evacuation and sterilisation in view of cardiac disease. Previous medical records revealed that patient was a diagnosed case of Peripartum Cardiomyopathy since 3 years, during first pregnancy, and had then presented with breathlessness on exertion; generalised oedema; an ejection fraction of 38\%; and global hypokinesia on echocardiography and her symptoms had improved with Digoxin, Aspirin, Spironolactone, Ramipril.

Currently, since past 6 months she is on Tab. Aspirin $75 \mathrm{mg}$ OD only. Her past history did not reveal any alcohol abuse or the use of $\beta$-adrenergic agonists, viral infection. She was also 
diagnosed with peripartum seizures 3 years back, and is on Sodium Valproate $300 \mathrm{mg}$ OD since then. She is also on Tab. Folic acid $5 \mathrm{mg}$ OD. Last seizure attack was 3 months back.

On conceiving again, she was advised by the cardiologist to terminate the pregnancy. During this pregnancy, she had breathlessness only on severe exertion (NYHA Class II). Her ECG showed T inversion in lead I, II, III, aVF, V4, V5, V6 with Poor R wave progression; echocardiography revealed $47 \%$ ejection fraction, akinetic distal IVS, LV apical aneurysm, while the other investigations were normal.

The patient and relatives were explained about the high risk and informed consent was taken.

Preoperatively, injection Amoxicillin with clavulanic acid $1.2 \mathrm{~g}$ and injection Gentamycin $80 \mathrm{mg}$ IV. Patient required a $15^{\circ}$ head up position while supine. She was pale, tachypnoeic with respiratory rate $20-25$ breaths per minute. On auscultation, lungs were clear. Her airway was Mallampati grade I. The patient's baseline blood pressure (BP) and heart rate were $110 / 70 \mathrm{mmHg}$ and 118 /minute, respectively.

In the OT, $18 \mathrm{G}$ intravenous cannula was secured, she was preloaded with $250 \mathrm{~mL}$ of Ringer lactate. Intraoperative monitoring included continuous ECG, non-invasive blood pressure (NIBP), blood oxygen saturation $\left(\mathrm{SpO}_{2}\right)$. After taking all aseptic precautions, five milligrams of $0.5 \%$ heavy bupivacaine and $25 \mathrm{mcg}$ of Fentanyl citrate were administered intrathecally through the L3-L4 interspace in a single atraumatic attempt and a sensory block, up to the T8 level, was achieved after five minutes. Inj. Diazepam $2 \mathrm{mg}$ was used for sedation.

Intraoperative pulse rate ranged from 116-134 per minute, BP $110 / 70$ to $90 / 60 \mathrm{~mm}$ of $\mathrm{Hg}$. SpO2 98-99\%, ECGsinus tachycardia. $600 \mathrm{~mL}$ of crystalloids administered and urine output was $70 \mathrm{~mL}$ intraoperatively. Total duration of surgery was $20 \mathrm{~min}$. The patient remained haemodynamically stable intraoperatively and postoperatively.

Patient was shifted to ICU for observation and further management with instructions to keep the patient in propped up position and Oxygen through VentiMask. Postoperative course of the patient was uneventful, and she was discharged on fourth day.

\section{DISCUSSION}

PPCM is a rare form of dilated cardiomyopathy associated with pregnancy. It was first reported in the $19^{\text {th }}$ century by Ritchie.[5] It is defined as "the onset of acute heart failure in the last trimester or early postpartum period in the absence of infectious, metabolic, toxic, ischemic or valvular cause of myocardial dysfunction." Although the exact aetiology is unknown, a multitude of causes including nutritional deficiencies, hormonal effects, small vessel coronary artery abnormality, toxaemia, maternal immunologic response to foetal antigen or myocarditis have been proposed. Most of the patients are asymptomatic with cardiomegaly and minimal cardiovascular symptoms and later in the course present with cardiac failure, where the mortality rate is high.

The predictors of poor prognosis are an ejection fraction of less than 0.25 (as seen on Echo, during the acute presentation of heart failure), left ventricular end diastolic dilatation, a hypokinetic left ventricle, the presence of mitral and tricuspid regurgitation.[6]

The main management goals in these patients under any kind of anaesthesia include:

1. Maternal and foetal safety.
2. Avoidance of myocardial depression and maintaining stable haemodynamics.

3. Maintaining uteroplacental adequacy.

4. Maintaining normovolaemia and preventing increase in the ventricular afterload, preload optimisation, and increased contractility of the heart. Analgesia must be adequate to prevent an increase in blood pressure and heart rate.

Seizure is a symptom characterised by a paroxysmal and transitory disturbance of cerebral functions, which develop suddenly, cease spontaneously and exhibit a conspicuous tendency to recurrence. Incidence of Seizures is $0.3-0.6 \%$ in pregnant women.[7]

During pregnancy there is no reliable source which allows prediction of an individual's course of epilepsy except the pregestational fit frequency. Knight and Rhind found that $45 \%$ of women with epilepsy had an increased frequency of seizures, $5 \%$ experienced a decrease and $50 \%$ had no change. A review of the literature revealed increase in seizure frequency from $23 \%$ to $75 \%$ amongst pregnant women who had epilepsy[8]. Women, who remain seizure-free for 9 months preceding pregnancy, are at $20-40 \%$ risk of exacerbation of seizures during pregnancy. And those who convulse at least once monthly usually worsen during pregnancy. Folic acid has been shown to decrease the incidence of neural tube defects in women on Antiepileptic drugs (AEDs). All women on AEDs should be prescribed folic acid in the dose of $5 \mathrm{mg} /$ day which should be started pre-conceptionally and continued throughout pregnancy.

In this type of cases, PPCM with seizure disorder is a major challenge and anaesthesiologists have a major role. Interventions are required to decrease both preload and afterload as well as to improve cardiac contractility and prevent precipitation of seizures preoperatively and postoperatively.

Both general and regional anaesthesia have been used

In general anaesthesia, the stress of laryngoscopy and intubation coupled with the myocardial depressant effects of anaesthetics are not beneficial for these patients. There are reports of severe embolic stroke [1] and cardiac failure [9] with general anaesthesia. McIndoe et al. described a previously asymptomatic parturient with PPCM who presented with a cardiac arrest at induction of general anaesthesia for emergency caesarean section. [9] Similarly, Wake et al. reported cardiac arrest immediately after the start of surgical procedure during emergency caesarean section under general anaesthesia in a patient with PPCM.[10]

Regional anaesthesia (RA) also produces vasodilatation, causing a fall in preload and afterload on the left ventricle without improving contractility which may be beneficial in a situation of poor ventricular function, where no outflow tract obstruction is present, which prevents thromboembolic events and decreases the epinephrine/norepinephrine levels. RA, however, may be contraindicated in an anticoagulated patient. Although the isolated use of Aspirin does not increase the risk of spinal haematoma and does not represent a contraindication to neuraxial blocks.

Use of an intrathecal opioid prevents haemodynamic instability associated with spinal anaesthesia by decreasing the dose requirement of bupivacaine. 
Although anaesthetists are apprehensive, but there are case reports where low dose spinal block has been successful and can be tried.[11] As the anticipated duration of Suction Evacuation with Sterilisation is less, we managed the case with low dose Bupivacaine spinal block without any complications as there was no need to prolong the duration of block. It also has a favourable neurotoxic profile compared to Lignocaine. Diazepam, a Benzodiazepine, increases the seizure threshold and has low BP and HR variation.[12]

\section{CONCLUSION}

PPCM is a disease associated with high morbidity and mortality. Principles of therapy remain more or less same as that in heart failure due to any cause. Additionally, patient was a known case of seizure disorder which increases the risk furthermore. Although our patient had PPCM but because of appropriate medical treatment she was asymptomatic preoperatively and was managed successfully with spinal anaesthesia. Patients like these, of cardiomyopathy with no features of congestive cardiac failure can be managed successfully with low dose of local anaesthetic with opioid. Our case report highlights the same and signifies the importance of spinal anaesthesia in such patients.

\section{ACKNOWLEDGEMENT}

I acknowledge with thanks the support extended by Dr. Vithal K Dhulkhed, Professor \& HOD of Anaesthesiology, Postgraduate students and Research Committee, KIMS, Karad.

\section{REFERENCES}

1. Shiva K, Fett J, Elkayam U. Peripartum cardiomyopathy. Lancet 2006;368(9536):687-93.

2. Tanganelli P, Regesta G. Epilepsy, pregnancy and major birth anomalies: an Italian prospective, controlled study. Neurology 1992;42(4 suppl 5):89-93.
3. Costa AL, Lopes-Cendes I, Guerreiro CA. Seizure frequency during pregnancy and the puerperium. Inter J Gynaecol Obstet 2005;88(2):148-9.

4. Pearson GD, Veille JC, Rahimtoola S, et al. Peripartum cardiomyopathy: national heart, lung and blood institute and office of rare diseases (National Institute of health) workshop recommendations and review. JAMA 2000;283(9):1183-8.

5. Ritchie C. Clinical contributions to the diagnosis and treatment of certain chronic diseases of the heart. Med Surg J 1849;2:333.

6. William G, Fuster V. Idiopathic dilated cardiomyopathy. New Engl J Med 1994;331(23):1564-75.

7. Knight AH, Rhind EG. Epilepsy and pregnancy: a study of 153 pregnancies in 59 patients. Epilepsia 1975;16(1):99110.

8. Yerby MS. Pregnancy and epilepsy. Epilepsia 1991;32(Supple 6):S51-9.

9. Wake K, Takanishi T, Kitajima T, et al. Cardiac arrest during emergency caesarean section due to peripartum cardiomyopathy. Masui 2003;52(10):1089-91.

10. McIndo AK, Hammond EJ, Babington PC. Peripartum cardiomyopathy presenting as a cardiac arrest at induction of anaesthesia for emergency caesarean section. Brit J Anaesth 1995;75(1):97-101.

11. Brown G, O'Leary M, Douglas I, et al. Perioperative management of a case of severe peripartum cardiomyopathy. Anaesth Intens Care 1992;20(1):80-3.

12. Longius EN, Emmanuel E, Dabota BY. Spinal anaesthesia for peripartum cardiomyopathy. Update Anaesth 2010;26:43-5. 